\title{
Current-Modulation-based on-line Resonance Tuning Strategy for Linear Generator Drives
}

\author{
Min Zhang, Alexey Bodrov, Roger Shuttleworth, Matteo F. lacchetti, Senior Member, IEEE
}

\begin{abstract}
Linear Generators (LGs) are frequently used for energy harvesting with free-piston Stirling engines, thermoacoustic engines and wave energy converters.

This paper presents a control strategy to track and maintain LG resonance conditions in real time. The algorithm is based on the LG response to a low-frequency amplitude modulation of the current component in phase with the instantaneous position (d-axis). The averaged product of modulated airgap power and modulation signal is fed into a controller to adjust the $d$-axis current and restore resonance. The use of airgap power instead of dc power improves resonance tracking accuracy and eliminates steady-state low-frequency stroke oscillations. The paper presents a full theoretical analysis providing accurate steady-state and small-signal models for control synthesis. The broad experimental validation included in the paper proves that the control is able to restore resonance even when the force-source introduces significant additional mechanical impedance.
\end{abstract}

Index Terms- PM Linear Generator, Linear Alternator, Resonance Tracking, Thermoacoustic Electric Generator, Wave energy Converter, Energy Harvesting.

\section{NOMENCLATURE}

$c \quad$ damping coefficient.

$F \quad$ driving force.

$i, I \quad$ instantaneous current, current amplitude.

$I_{\varepsilon} \quad$ low-frequency current modulation magnitude.

$i_{L M} \quad$ linear motor instantaneous current.

$k, k_{E} \quad$ stiffness coefficient, EMF constant.

$m \quad$ LG moving mass.

$p_{d c}, p_{\text {gap }}$ dc power, airgap power.

$x, X \quad$ instantaneous position, position amplitude.

$X_{1} \quad$ steady-state $1^{\text {st }}$-harmonic position amplitude.

$X_{\varepsilon} \quad$ low-frequency position modulation magnitude.

$\varepsilon \quad$ "out of resonance" error.

$\varphi_{1} \quad$ phase-shift between position and force.

Manuscript received August 05, 2019; revised November 24, 2019 and January 28, 2020, accepted February 19, 2020. The work reported in this article was supported by the U.K. Engineering and Physical Sciences Research Council, through project HARP2: Holistic Approach to the Design of Efficient Heat Recovery Systems for Electrical Power Production, under Grant EP/R02328X/1. (Corresponding author Dr. Matteo F. lacchetti).

M. Zhang, A. Bodrov, R. Shuttleworth and M. F. lacchetti are with the Department of Electrical and Electronic Engineering. The University of Manchester, Manchester, M13 9PL, U.K. (e-mail: min.zhang-9, alaxey.bodrov, roger.shuttleworth, matteo.iacchetti @manchester.ac.uk). $\omega, \omega_{m 0} \quad$ angular frequency, mechanical resonance frequency.

\section{Subscripts and superscripts}

$d, q \quad$ in-phase, in-quadrature axis (with position).

$\varepsilon \quad$ low-frequency modulation.

$0 \quad$ quiescent point for linearization.

*,\# reference value, PI controller output.

\section{INTRODUCTION}

$\mathrm{R}$ ECIPROCATING Linear Generators (LGs) allow power conversion from pressure waves (via a piston) directly into electricity without need of complex mechanisms such as crank-shaft and con-rods. They are a vital component of direct-drive Sea Wave Energy Converters (SWECs) [1], FreePiston Stirling Engines (FPSEs) [2], and Thermoacoustic Engines (TAEs) [3]. In many of these systems, the LG plunger and piston assembly is attached to the stator frame by springs so as to create a resonant mechanical system at the pressure wave frequency, thereby counteracting inertia forces. The spring stiffness design can also take into account the reactive component of pressure source impedance in order to force resonance, which is a necessary condition for impedance matching [4]. In a LG, the current should ideally be controlled to be in quadrature with instantaneous position, so as to maximize generated power [5]. The control implementation requires either position measurement [6] or estimation of Electromotive Force (EMF) [7]. Design and construction tolerances and changes in pressure wave frequency, however, may cause the LG to work away from resonance, destroying the impedance matching and reducing stroke amplitude and power output. Cogging is another detrimental factor which can affect the resonance frequency. Cogging can be alleviated with core-less design [8], at the expense of a larger PMs.

In Linear Motors (LMs), the operating frequency is a free variable which can be adjusted automatically to enforce resonance conditions. The implementation of this concept requires a resonance detection block and a controller to adjust frequency until instantaneous position and current signals are in quadrature. Resonance detection relies on monitoring either zero crossings of current and instantaneous position [9] (or velocity [10]), or averaged product of position $\times$ current $[11]$. The sensitivity to system parameters can be addressed by using on-line parameter estimation [12]. As an alternative, resonance frequency can be indirectly tracked with a search algorithm maximizing input electrical power [13].

In energy harvesting systems, however, frequency is set by the "prime mover" (e.g. SWEC, FPSE, or TAE) and the LG 
has no direct control of frequency, so resonance conditions cannot be maintained as easily as in LM drives. However, the LG control can regulate the current component in phase with instantaneous position ( $d$-axis current) in order to add or subtract "electronic" (or virtual) stiffness to the mechanical stiffness provided by springs [14]-[15]. The method adjusts the $L G$ resonance frequency to match the operating frequency dictated by the prime mover, thereby maintaining resonance conditions. Controlling the $d$-axis current requires a Voltage Source Inverter (VSI) and cannot be obtained only via passive rectification and boost converter [25]. L-C networks on the stator terminals can also add electronic stiffness but do not offer flexible regulation [17]. Clearly, the additional current demand for electronic stiffness will increase the VSI and LG ratings [16]. Output-power versus power-factor maps can be used to identify a trade-off between output power maximization and LG-VSI rating minimization [26].

The required zero phase-shift between excitation force and velocity can be achieved also by shaping the LG electromagnetic force every quarter cycle [19], so as to transiently slow down or accelerate the translator and control the phase of the first harmonic of the velocity waveform. However, this method introduces force harmonics which are potentially harmful for TAE and FPSE operation.

Although electronic-stiffness control [15] does not cause distortion, the implementation needs accurate knowledge of LG mechanical stiffness and mass, along with real-time frequency estimation to determine the required electronic stiffness. This requirement introduces sensitivity to LG mechanical parameters and does not allow the system to cope with the driving-force source impedance, which is often unknown. Perturb and observe algorithms can be adopted to eliminate parameter sensitivity and better deal with the wide wave spectrum in SWECs [18]. As a drawback, these methods do not offer an easy way to tune perturbation and correction step magnitudes, apart from trial and error [24]. In addition, they can lead to persistent stroke amplitude fluctuations.

In order to overcome these limitations, in [21] the electronic stiffness tuning concept was coupled with an automatic resonance detection scheme which does not require knowledge of system parameters. Resonance is detected by analyzing LG output power modulation caused by a permanent lowmagnitude, quasi-static modulation purposely introduced into the amplitude of the current component in phase with position. The averaged product of modulated power and current envelopes is fed into a Proportional-Integral (PI) controller to adjust $d$-axis current level and restore resonance.

The scheme in [21], however, does not take into account Joule and VSI losses, which cause inaccurate resonance tracking, especially in small LGs with high stator resistance. Furthermore, the steady-state and small-signal models presented in [21] are inaccurate and produce unreliable controller gain settings. Finally, [21] only provided simulation results in ideal force-driven conditions, with no experimental validation and no consideration for practical scenarios where the pressure wave source may introduce significant unknown impedance. This paper presents fundamental novelties with respect to the previous work:

1) Implementation based on air-gap power instead of $\mathrm{dc}$ power, in order to improve resonance tracking accuracy.

2) New accurate steady-state and small-signal models to fully address the control synthesis, whereas the model in [21] yields predictions affected by errors in excess of $80 \%$.

3) Thorough experimental validation in both force-driven conditions and more realistic scenarios, where the drivingforce source exhibits significant internal impedance.

The paper is structured as follows. Sections II and III describe the modelling and control principle for the LG. Section IV derives steady-state equations for low-frequency stroke and power modulation components. The small-signal model is subsequently derived in Section V. Finally, Sections VI and VII present extensive numerical and experimental validation on a test-rig comprising a LG designed for a TAE.

\section{MODELLING}

Fig. 1 shows the schematic of the LG and dc-link-tied VSI.

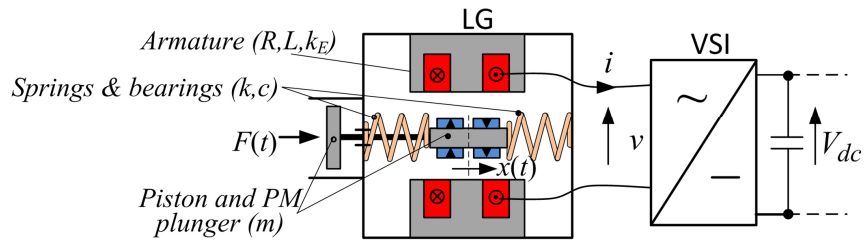

Fig. 1. PM LG and VSI interface.

In this paper, the $\mathrm{LG}$ is considered to be driven by a harmonic force of amplitude $F$ at angular frequency $\omega$. The mechanical impedance, however, may incorporate the pressure wave source impedance. The electromagnetic force $F_{e}=k_{E} i$ is produced by current $i$, which is controlled by the VSI. The equivalent circuit is given in Fig. 2(a).

The dynamic equations for the single-phase LG are:

$$
\begin{gathered}
m \ddot{x}+c \dot{x}+k x=F(t)-k_{E} i \\
\frac{d i}{d t}=\frac{1}{L}\left(k_{E} \dot{x}-R i-v\right)
\end{gathered}
$$

Where $m, c$ and $k$ are the mass, damping and stiffness, $R$ and $L$ are the resistance and inductance, and $k_{E}$ is the EMF constant.
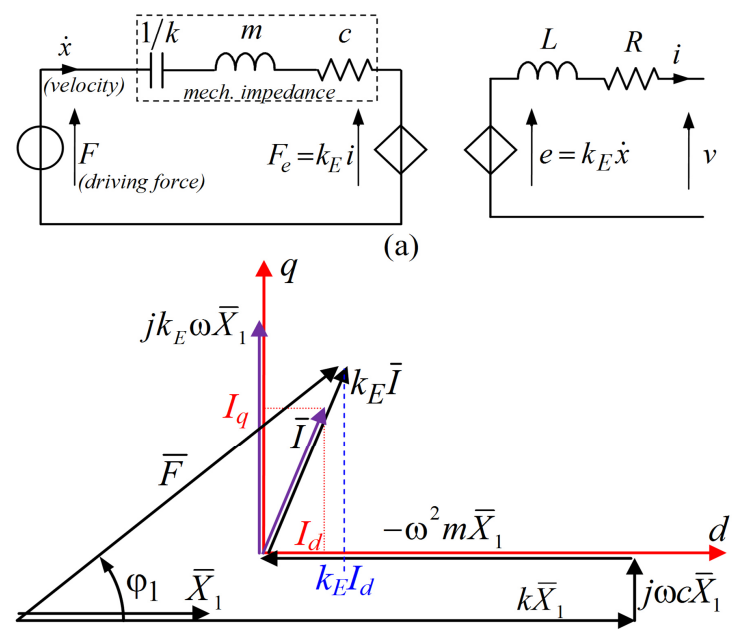

(b)

Fig. 2. Coupled mechanical and electrical equivalent circuit (a). Steady-state mechanical and electrical phasor diagram (b). 
Fig. 2(b) shows the steady-state phasor diagram at a generic frequency below resonance (position lags force by angle $\varphi_{1}$ ). Axes $d$ and $q$ are in-phase and in-quadrature with steady-state instantaneous position $x(t)=X_{1} \cos \omega t$. Capital letters denote steady-state values and upper bars mean phasors. In this paper, $d-q$ modelling is used for steady-state analysis, and a currentdriven LG (via hysteresis current control) is considered. Therefore, the steady-state current can be written as

$$
i(t)=i_{d}(t)+i_{q}(t)=I_{d} \cos \omega t-I_{q} \sin \omega t .
$$

\section{CONTROL SCHEME}

Fig. 3 shows the full control scheme comprising a PhaseLocked-Loop (PLL) tracking position from a sensor, the current amplitude generation blocks, including the electronic stiffness tuning, and the hysteresis controller. The general structure follows the scheme in [21]. Unlike [21], however, Fig. 3 includes compensation for LG Joule and VSI losses in order to avoid inaccurate resonance tracking. The Linear Time-Invariant Enhanced PLL (LTI-EPLL) synchronizing the control with the prime-mover frequency is described in [23].

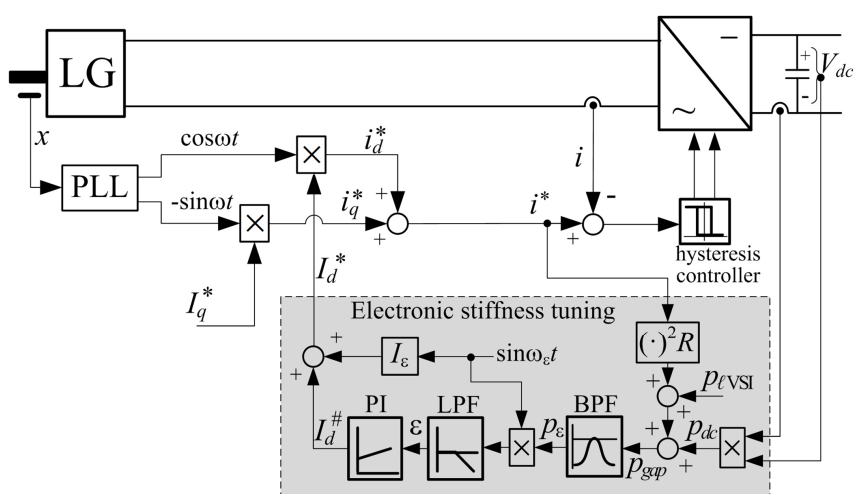

Fig. 3. LG control with improved automatic tuning of the electronic stiffness compensating for the LG and VSI losses.

The PLL generates in-phase and in-quadrature unityamplitude waveforms $\cos \omega t$ and $-\sin \omega t$. Once multiplied by appropriate amplitudes $I_{d}{ }^{*}$ and $I_{q}{ }^{*}$, they give the instantaneous current command " $i{ }^{*}(t)=i_{d}{ }^{*}(t)+i_{q}{ }^{*}(t)=I_{d}{ }^{*} \cos \omega t-I_{q}{ }^{*} \sin \omega t$ " to be forced into the LG winding via the VSI with hysteresis current control. Hysteresis control is preferred over PI control, because it allows more accurate tracking of sinusoidal reference signals typical of linear machines [22].

Reference amplitude $I_{q}{ }^{*}$ sets the power transfer level. Instantaneous current component $i_{d}$ in phase with position $x$ is used to synthesize an electronic stiffness and change the resonance frequency of the LG. The amplitude $I_{d}{ }^{*}$ of reference $i_{d}^{*}(t)$ comprises a relatively steady contribution $I_{d}{ }^{\#}$ (set by the electronic stiffness control), added to which is a small quasistatic perturbation term $I_{\varepsilon} \sin \omega_{\varepsilon} t$ at $\omega_{\varepsilon}<<\omega$. The perturbation produces a low-frequency modulation in stroke and airgap power. The resulting phase-shift between low-frequency stroke amplitude modulation and perturbation signals is shown in Fig. 4 for operation below, above and at resonance.

A quasi-static increase in stiffness over the first half-period increases the resonance frequency and leads to a corresponding decrease or increase in stroke amplitude, if the driving-force frequency is below or above the rated resonance value $\omega_{m 0}$ respectively. Consequently there is an almost $180^{\circ}$ or $0^{\circ}$ phase-shift between current modulation signal and modulated stroke (and thus airgap power envelope) as shown in Fig. 4(a) and 4(b) respectively. Conversely, when the system is operating at resonance, Fig. 4(c), any stiffness increase or decrease causes a drop in stroke and power: hence, the $\omega_{\varepsilon}$-harmonic in the stroke envelope disappears and is replaced by a second-order harmonic at $2 \omega_{\varepsilon}$.
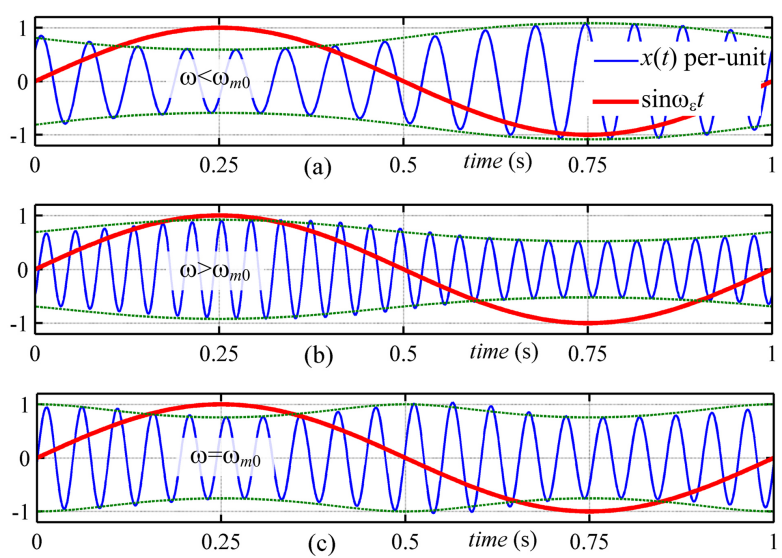

Fig. 4. Stroke modulation (blue/green) due to a stiffness perturbation (red) in the operation below (a), above (b), or at resonance (c) [21].

Quantity $p_{\varepsilon}$ in Fig. 3 denotes the modulating component in the airgap power $\left(p_{\text {gap }} \approx p_{d c}+R i^{* 2}+p_{\ell \mathrm{VSI}}\right)$ at low frequency $\omega_{\varepsilon}$, to be extracted with a Band-Pass Filter (BPF) tuned at $\omega_{\varepsilon}$. Once filtered by a Low-Pass Filter (LPF) with time constant $\tau>>2 \pi / \omega_{\varepsilon}$, the average product of modulated power $p_{\varepsilon}$ and perturbation $\sin \omega_{\varepsilon} t$ gives an "out-of-resonance error" $\varepsilon$. The error is fed to a PI controller (gains $k_{p}$ and $k_{i}$ ) which adjusts the amplitude $I_{d}$ \# of $d$-axis current component accordingly.

If $p_{\varepsilon}$ were based on $p_{d c}$ alone, it would be affected by extra terms at $\omega_{\varepsilon}$ due to the modulated LG Joule, $R i^{2}$, and VSI, $p_{\ell \mathrm{VSI}}$, losses via $I_{\varepsilon} \sin \omega_{\varepsilon} t$. These losses degrade the accuracy of resonance tracking. For this reason and unlike in [21], in Fig. 3 error $\varepsilon$ is calculated using airgap power instead of dc power.

The main application for the proposed control is resonance tracking in systems with pressure wave excitation characterized by slow dynamics such as TAEs.

\section{Steady-State Analytic Model}

\section{A. Approximate solution for modulated stroke}

The analytic approach in [21] translated the $d$ - and $q$-axis current amplitudes into additional equivalent stiffness and damping values to be added to $k$ and $c$. The $d$-axis current amplitude modulation resulted in a parametric (stiffness) excitation. Equivalent stiffness and damping were obtained by multiplying $d$ - and $q$-axis current amplitudes by instantaneous position and velocity formally normalized to their steady-state first-harmonic amplitudes $X_{1}$ and $\omega X_{1}$, then leading to the Mathieu-like equation (4). From here on, superscripts " **" and 
“\#” are dropped to simplify the notation.

$m \ddot{x}+\left(c+\frac{k_{E} I_{q}}{\omega X_{1}}\right) \dot{x}+\left(k+\frac{k_{E} I_{d}}{X_{1}}+\frac{k_{E} I_{\varepsilon} \sin \omega_{\varepsilon} t}{X_{1}}\right) x=F \cos \left(\omega t+\varphi_{1}\right)$

Amplitude $X_{1}$ refers to the steady-state operation for the unperturbed system (i.e. with $I_{\varepsilon}=0$ ). A quasi-static approximate solution of (4) was then derived in the form

$$
x(t) \cong X(t) \cos \omega t
$$

The modulated amplitude $X(t)$ was obtained from the wellknown solution of the one-degree-of-freedom vibrating system by assuming constant parameters and by perturbing $I_{d}$ in the final expression. Despite its appealing feature of lumping mechanical and electronic stiffness terms together, (4) is inaccurate and does not lend itself to the derivation of reliable steady-state and small-signal models (see Table II).

The approach presented in this paper assumes the same structure (5) for the solution, but obtains $X(t)$ by replacing $I_{d}$ with $I_{d}+I_{\varepsilon} \sin \omega_{\varepsilon} t$ in the solution for the steady-state equation of the unperturbed system (6), instead of (4).

$$
m \ddot{x}+c \dot{x}+k x=F \cos \left(\omega t+\varphi_{1}\right)-k_{E} I_{d} \cos \omega t+k_{E} I_{q} \sin \omega t
$$

Note that (6) enforces the orientation constraint for currents by multiplying $I_{d}$ and $I_{q}$ by unit waveforms " $\cos \omega t$ " and "-sin $\omega t$ ". Equation (6) is based on the following assumptions:

- The current amplitude modulation frequency $\omega_{\varepsilon}$ is much lower than the operating frequency $\omega$.

- The fast hysteresis control tracks $i^{*}$ instantaneously $\left(i \approx i^{*}\right)$.

- The dynamics of the PLL are ignored so that instantaneous orientation of $d$-axis with position is achieved.

Equation (6) is solved by replacing $\cos \omega t$ with $e^{j \omega t}$ and deriving the solution in the form $x(t)=X_{1} e^{j \omega t}$. After substitution and manipulation, amplitude $X_{1}$ is given by:

$$
X_{1}=-a_{1}+\sqrt{F^{2} / h-b^{2}},
$$

with

$$
\begin{gathered}
h=(\omega c)^{2}+\left(k-m \omega^{2}\right)^{2}, \\
a_{1}=k_{E}\left(\omega c I_{q}+\left(k-m \omega^{2}\right) I_{d}\right) / h, \\
b=k_{E}\left(\omega c I_{d}-\left(k-m \omega^{2}\right) I_{q}\right) / h .
\end{gathered}
$$

Replacing $I_{d}$ with $I_{d}+I_{\varepsilon} \sin \omega_{\varepsilon} t$ in (9)-(10) and then in (7) gives an approximate equation for modulated amplitude $X(t)$.

$$
X(t)=-a_{1}-a_{2} I_{\varepsilon} \sin \omega_{\varepsilon} t+\sqrt{\frac{F^{2}}{h}-\left(b+\frac{k_{E} \omega c I_{\varepsilon}}{h} \sin \omega_{\varepsilon} t\right)^{2}}
$$

With

$$
a_{2}=k_{E}\left(k-m \omega^{2}\right) / h .
$$

(4)) is that the modulated $d$-axis current $I_{d}+I_{\varepsilon} \sin \omega_{\varepsilon} t$ is replaced into the final solution of (6) - that is (7) - whereas in [21] term " $I_{d}+I_{\varepsilon} \sin \omega_{\varepsilon} t$ " appears only in the electronic stiffness definition using unperturbed stroke amplitude $X_{1}$.

\section{B. Stroke amplitude modulation}

Due to the radical in (11), the Fourier coefficients for harmonics of frequency $\omega_{\varepsilon}$ involve Elliptic integrals. However, approximate expressions can be derived as follows. Expanding the radicand and neglecting terms with $I_{\varepsilon}{ }^{2}$ gives

$$
\begin{gathered}
\sqrt{\frac{F^{2}}{h}-\left(b+\frac{k_{E} \omega c I_{\varepsilon}}{h} \sin \omega_{\varepsilon} t\right)^{2}} \approx \sqrt{\frac{F^{2}}{h}-b^{2}-\frac{2 b k_{E} \omega c I_{\varepsilon}}{h} \sin \omega_{\varepsilon} t} \\
=\sqrt{\frac{F^{2}}{h}-b^{2}} \sqrt{1-\frac{2 b k_{E} \omega c I_{\varepsilon}}{F^{2}-h b^{2}} \sin \omega_{\varepsilon} t} .
\end{gathered}
$$

With typical parameter values, the coefficient of $\sin \omega_{\varepsilon} t$ in the last term of (13) is much less than unity. Thus, approximation $(1+\delta)^{1 / 2} \approx 1+\delta / 2$ can be used for the radical in (13), leading to

$$
X(t) \cong-a_{1}-a_{2} I_{\varepsilon} \sin \omega_{\varepsilon} t+\sqrt{\frac{F^{2}}{h}-b^{2}}\left(1-\frac{b k_{E} \omega c I_{\varepsilon}}{F^{2}-h b^{2}} \sin \omega_{\varepsilon} t\right) .
$$

Equation (14) gives immediately the wanted amplitude $X_{\varepsilon}$ of the stroke modulating term at frequency $\omega_{\varepsilon}$ :

$$
X_{\varepsilon}=\left(-a_{2}-\sqrt{\frac{F^{2}}{h}-b^{2}} \frac{k_{E} b \omega c}{F^{2}-h b^{2}}\right) I_{\varepsilon} .
$$

The mean value of (14) over $2 \pi / \omega_{\varepsilon}$ period is the un-modulated stroke amplitude $X_{1}$ with constant $I_{d}$, as from (7). Finally, (15) is rewritten in terms of $I_{d}$ and $I_{q}$ by using (8)-(10) and (11).

$X_{\varepsilon}=-\frac{k_{E} I_{\varepsilon}}{h}\left(k-m \omega^{2}+\frac{c \omega k_{E}\left(\omega c I_{d}-\left(k-m \omega^{2}\right) I_{q}\right)}{\sqrt{F^{2} h-k_{E}^{2}\left(\omega I_{d}-\left(k-m \omega^{2}\right) I_{q}\right)^{2}}}\right)$

When the system is not operating at mechanical resonance (i.e. $\omega \neq \omega_{m 0}=\sqrt{k / m}$ ), the control should adjust $I_{d}$ in order to restore resonance at any operating frequency $\omega$. The required $I_{d}$ value should compensate for the "stiffness defect" $\left(\omega^{2} m-k\right)$, so that $k_{E} I_{d}=\left(\omega^{2} m-k\right) X_{10}$ with $X_{10}=\left(F-k_{E} I_{q}\right) /(c \omega)$ is the stroke amplitude at resonance (limited only by damping $c$ ). Therefore, the $I_{d}$ value restoring resonance at generic $\omega$ is

$$
I_{d \mathrm{res}}=-\frac{F-k_{E} I_{q}}{k_{E} c \omega}\left(k-m \omega^{2}\right) .
$$

When the system operates at the mechanical resonance frequency $\omega_{m 0}=\sqrt{k / m},(17)$ yields the expected $I_{d \text { res }}=0$, and (16) gives $X_{\varepsilon}=0$. By placing (17) into (16) it is verified that $X_{\varepsilon}=0$ at a generic frequency $\omega$ providing that $I_{d}=I_{d}$ res (17). This proves that $X_{\varepsilon}$ (16) can be used to detect both "mechanical" and "restored" resonance conditions.

The fundamental difference to the approach in [21] (based on 


\section{Airgap power modulation and "out-of-resonance" error}

The control scheme adopts dc power measurements, adding LG winding and VSI losses to obtain the airgap power. For the theoretical analysis, airgap power modulation component $p_{\varepsilon}$ at $\omega_{\varepsilon}$ can be estimated from airgap power $p_{\text {gap }}=k_{E} \dot{x} i$, neglecting low-frequency variations in inductor energy. Deriving (5) with respect to time and ignoring small term $\omega_{\varepsilon} X_{\varepsilon}$ gives

$$
\dot{x} \cong-\omega\left(X_{1}+X_{\varepsilon} \sin \omega_{\varepsilon} t\right) \sin \omega t .
$$

The steady-state current synchronized to the position signal through the EPLL and fast hysteresis current control is:

$$
i \cong\left(I_{d}+I_{\varepsilon} \sin \omega_{\varepsilon} t\right) \cos \omega t-I_{q} \sin \omega t .
$$

The resulting product of $k_{E} \dot{x} i$, from (18) and (19) generates many harmonics with frequencies much higher than $\omega_{\varepsilon}$. Since $\omega_{\varepsilon}<<\omega$, factor $\sin \omega_{\varepsilon} t$ can be treated as a modulating term. The BPF acting on the instantaneous airgap power returns a moving average of the high frequency terms. Furthermore, second-order factor $X_{\varepsilon} I_{\varepsilon}\left(\sim I_{\varepsilon}^{2}\right.$ due to (16)) can be neglected. Finally, the moving average of $p_{\text {gap }}$ over a period $2 \pi / \omega$ is

$$
p_{\text {gap ave }}(t)=\frac{k_{E} \omega}{2} X_{1} I_{q}+\frac{k_{E} \omega}{2} X_{\varepsilon} I_{q} \sin \omega_{\varepsilon} t
$$

The BPF blocks the mean value of (20) and returns the lowfrequency amplitude term $p_{\varepsilon}=(1 / 2) k_{E} \omega X_{\varepsilon} I_{q} \sin \omega_{\varepsilon} t$. The average of $p_{\varepsilon}$ over a $2 \pi / \omega_{\varepsilon}$ period is outputted by the LPF and represents the "out of resonance" error $\varepsilon$.

$$
\varepsilon=\frac{k_{E} \omega X_{\varepsilon} I_{q}}{4}
$$

\section{SMALL-SIGNAL ANALYSIS}

The small-signal model of the $I_{d}$ control is derived by perturbing the steady-state amplitudes and then introducing the LPF as in Fig. 3. The approach can model the slow dynamics of amplitudes in single-phase systems and is justified by the presence of a LPF with $\tau>>1 / \omega_{\varepsilon}$. The BPF and the product blocks in Fig. 3 form a demodulator and provide the airgap power component at $\omega_{\varepsilon}$. Therefore, this paper assumes an ideal demodulator represented by unity gain.

The linearization is performed around a quiescent point (subscript "0") with generic frequency $\omega_{0}$ and $q$-axis current amplitude $I_{q 0}$, but assuming "restored" resonance conditions through $I_{d 0}=I_{d \mathrm{res} 0}$ from (17), so $F=c \omega_{0} X_{10}+k_{E} I_{q 0}$. The perturbation (symbol " $\Delta$ ") of (16) gives:

$$
\Delta X_{\varepsilon}=\left.\frac{\partial X_{\varepsilon}}{\partial I_{d}}\right|_{0} \Delta I_{d}+\left.\frac{\partial X_{\varepsilon}}{\partial I_{q}}\right|_{0} \Delta I_{q}+\left.\frac{\partial X_{\varepsilon}}{\partial \omega}\right|_{0} \Delta \omega .
$$

The partial derivatives of (16) at the quiescent point are:

$$
\begin{gathered}
\left.\frac{\partial X_{\varepsilon}}{\partial I_{d}}\right|_{0}=-\frac{k_{E}^{2} I_{\varepsilon}}{c \omega F} \quad,\left.\quad \frac{\partial X_{\varepsilon}}{\partial I_{q}}\right|_{0}=\frac{k_{E}^{2} I_{\varepsilon}}{c^{2} \omega^{2} F}\left(k-m \omega_{0}^{2}\right), \\
\left.\frac{\partial X_{\varepsilon}}{\partial \omega}\right|_{0}=\frac{k_{E} I_{\varepsilon}}{c^{2} \omega^{3} F}\left(F-k_{E} I_{q 0}\right)\left(k+m \omega_{0}^{2}\right) .
\end{gathered}
$$

At the quiescent point, $X_{\varepsilon 0}$ is zero so the linearization of (21) is

$$
\Delta \varepsilon=\frac{k_{E} \omega_{0} I_{q 0}}{4} \Delta X_{\varepsilon}
$$

Fig. 5 shows the final block diagram of the small-signal model for the automatic resonance-tuning mechanism. The structure and gains of Fig. 5 are quite different from those derived in [21] with the inaccurate model stemming from use of (4).

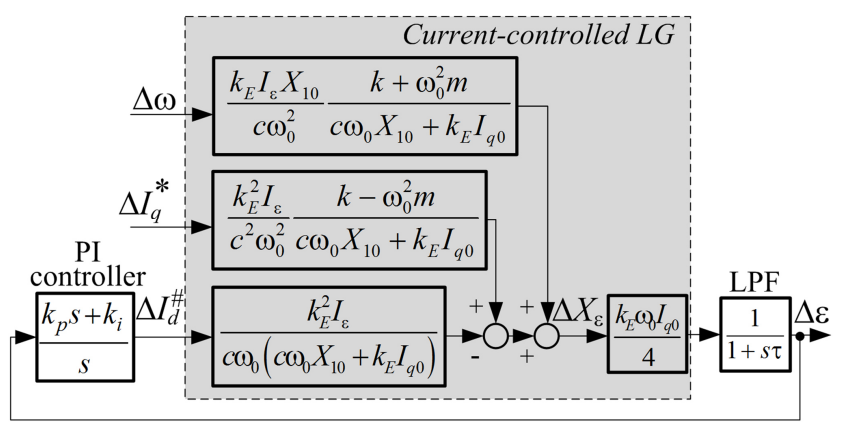

Fig. 5. Small-signal block diagram of the resonance tuning mechanism.

\section{Simulation Results}

A detailed Simulink model was built, comprising LA dynamic equations (1)-(2), the EPLL [23], the control scheme in Fig. 3 and a switching-function-based VSI. Simulations were carried out mainly to validate the steady-state and smallsignal models in Sections IV and V. The test-rig parameters in Table I were adopted for simulation: $m, c$ and $k$ values were doubled to represent the test rig with two identical, rigidlycoupled machines. The hysteresis controller band is $0.05 \mathrm{~A}$. The magnitude and frequency of $d$-axis current modulation are $I_{\varepsilon}=0.12 \mathrm{~A}$ and $\omega_{\varepsilon}=2 \pi \times 0.5 \mathrm{rad} / \mathrm{s}(0.5 \mathrm{~Hz})$ respectively. The BPF in Fig. 3 is a cascade of two identical $2^{\text {nd }}$-order resonant blocks with transfer function $2 \zeta_{\varepsilon} \omega_{\varepsilon} s /\left(s^{2}+2 \zeta_{\varepsilon} \omega_{\varepsilon} s+\omega_{\varepsilon}{ }^{2}\right)$ and damping ratio $\zeta_{\varepsilon}=4$. The first-order LPF time-constant $\tau$ is 10s. The PI controller synthesis was based on the model in Fig. 5: gains are set to $k_{p}=0.212 \mathrm{~V}^{-1}$ and $k_{i}=0.028(\mathrm{~V} \cdot \mathrm{s})^{-1}$ to achieve a settling time of $90 \mathrm{~s}$.

TABLE I: TEST-RIG PARAMETERS

\begin{tabular}{c|c}
\hline Armature resistance $R=2.4 \Omega$ & Rated Stroke $X_{M}=3.5 \mathrm{~mm} \mathrm{pk}$ \\
\hline Armature inductance $L=0.072 \mathrm{H}$ & Rated current $I_{M}=3 \mathrm{~A} \mathrm{pk}$ \\
\hline EMF constant $k_{E}=49.73 \mathrm{~V} \cdot \mathrm{s} / \mathrm{m}$ & DC load resistor $500 \Omega$ \\
\hline Plunger mass $m=0.79 \mathrm{~kg}$ & Rated freq. $37.3 \mathrm{~Hz}\left(\omega_{m 0}=234.4 \mathrm{rad} / \mathrm{s}\right)$ \\
\hline Stiffness $k=43.4 \mathrm{kN} / \mathrm{m}$ & Damping coefficient $c=14.9 \mathrm{~N} \cdot \mathrm{s} / \mathrm{m}$ \\
\hline
\end{tabular}

TABLE II: STEADY-STATE MOdEL VALIDATION AND COMPARISON TO [21]

\begin{tabular}{c|c|c|c|c|c|c}
\hline \multicolumn{5}{c}{$\begin{array}{c}\text { operating } \\
\text { conditions }\end{array}$} & \multicolumn{5}{c}{ Resulting $X_{\varepsilon}(\mathrm{mm})$} \\
\hline$\omega / \omega_{m 0}$ & $I_{d}{ }^{*}(\mathrm{~A})$ & Simulation & Eq. (11) in [21] & error \% & Eq. (16) & error \% \\
\hline \multirow{3}{*}{0.95} & 0.5 & -0.222 & -0.062 & 72.1 & -0.227 & 2.3 \\
\cline { 2 - 7 } & 0 & -0.140 & -0.048 & 65.7 & -0.144 & 2.9 \\
\cline { 2 - 7 } & -1 & 0.266 & 0.053 & 80.1 & 0.294 & 10.5 \\
\hline \multirow{4}{*}{1} & 0.5 & -0.172 & -0.048 & 72.1 & -0.181 & 5.2 \\
\cline { 2 - 7 } & 0 & 0.0015 & 0 & - & 0 & - \\
\cline { 2 - 7 } & -0.5 & 0.172 & 0.048 & 72.1 & 0.181 & 5.2 \\
\hline \multirow{3}{*}{1.05} & 1 & -0.258 & -0.050 & 80.6 & -0.280 & 8.5 \\
\cline { 2 - 7 } & 0 & 0.131 & 0.046 & 64.9 & 0.136 & 3.8 \\
\cline { 2 - 7 } & -0.5 & 0.193 & 0.056 & 71.0 & 0.207 & 7.3 \\
\hline
\end{tabular}


Table II compares simulated (benchmark) and theoretical values of steady-state amplitude modulation $X_{\varepsilon}$ caused by $I_{\varepsilon}=0.12 \mathrm{~A}$, under different operating frequencies $\omega$ (below, at, and above $\left.\omega_{m 0}\right)$ and $d$-axis current amplitudes $I_{d}{ }^{*}$, and with reference $q$-axis current amplitude $I_{q}{ }^{*}$ set at 2A. Equation (11) in [21] and (16) are both assessed. To have a persistent stroke modulation, simulations are run in open-loop, by disabling resonance tracking and $I_{d}{ }^{*}$ adjustment. Therefore, simulated $X_{\varepsilon}$ values can be used as a benchmark for both (11) in [21] and (16). Table II shows that (11) in [21] is inaccurate and may underestimate $X_{\varepsilon}$ with more than $80 \%$ error. Conversely, in the worst case scenario, formula (16) gives about $10 \%$ error, thereby validating the soundness of the analysis in Section IV.

Tests during the commissioning of the test-rig showed that the mechanical resonance frequency in the coupled LM-LG system $(36.5 \mathrm{~Hz}$, curve with markers "o" in Fig. 8) was slightly below the value in Table I $(37.3 \mathrm{~Hz})$. Therefore, in simulating the closed-loop response, the $k$ value was adjusted to $2 \times 41.5 \mathrm{kNs} / \mathrm{m}$, so as to allow direct comparison to the test in Fig. 12. Fig. 6 shows the response to a step change in the driving-force frequency from $36.5 \mathrm{~Hz}$ (resonance) to $38.5 \mathrm{~Hz}$ when the system is operating with $I_{q 0}=2 \mathrm{~A}$.
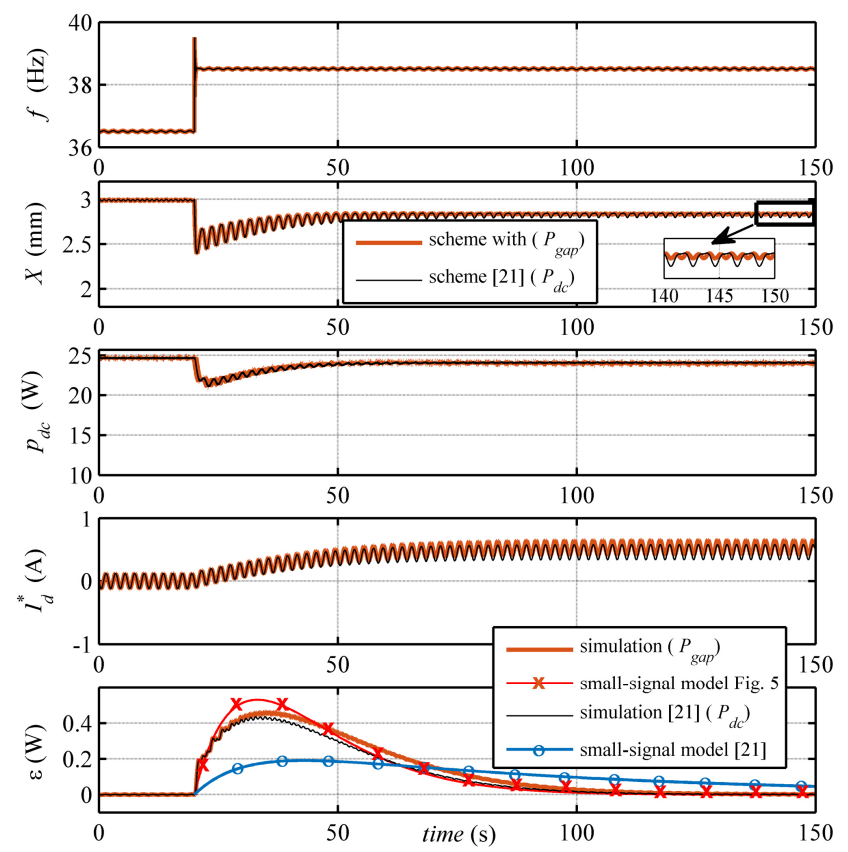

Fig. 6. Simulated response to a step in the operating frequency

Both $p_{d c}$-based scheme [21] (thin black trace) and $p_{g a p}$-based scheme (thick brown trace) are compared. After the step, the PI controller adjusts amplitude $I_{d}{ }^{*}$ until stroke amplitude $X$ is restored, so that the system operates at resonance at the new frequency. Due to the slight increase in frequency, damping losses increase and the final stroke amplitude decreases slightly. Fig. 6 confirms that at steady-state with the $p_{\text {gap }}$-based scheme there are no oscillations at $\omega_{\varepsilon}$ in amplitude $X$, and only small residual oscillations at $2 \omega_{\varepsilon}$. The final $I_{d}{ }^{*}$ mean-value matches with prediction $I_{d \mathrm{res}}=0.53$ A by (17). Finally, the bottom subplot shows the error $\varepsilon$ response compared to the step response based on the transfer function $\Delta \varepsilon / \Delta \omega$ from the diagram in Fig. 5. The mismatch in the response peak value is $\approx 20 \%$, but the overall accuracy is adequate for design purposes. While the implementation in [21] uses $p_{d c}$, the small-signal model in [21] neglects losses and is based on $p_{\text {gap }}$. The step response of transfer function $\Delta \varepsilon / \Delta \omega$ derived in [21] can therefore be directly compared with the $\varepsilon$ trends for the $p_{\text {gap }}{ }^{-}$ based scheme in the bottom subplot in Fig. 6. $\Delta \varepsilon / \Delta \omega[21]$ (markers "o") shows a sluggish response, due to the underestimated plant gains from the inaccurate $X_{\varepsilon}$ expression. The bottom subplot in Fig. 6 confirms the superior accuracy of the proposed theoretical framework over the one in [21]. Further validation and comparison are provided in Section VII.

\section{EXPERIMENTAL VALIDATION}

The automatic resonance tuning strategy was validated on the test-rig shown in Fig. 7, comprising two identical singlephase linear machines rated to $60 \mathrm{VA}$, with the parameters in Table I. The machines are back-to-back connected: one is used as a motor (LM) and the other as a generator (LG). The LG was interfaced to the Texas Instruments (TI) TMDXIDDK379D VSI (VSI1) connected to a stand-alone dc load resistor. The instantaneous position is measured using a Linear Variable Differential Transformer (LVDT). The overall control was implemented in a floating point Microcontroller TI Delfino TMS320F28379D (DSP1). The EPLL and PI controller were digitalized with the Euler method using a $100 \mu$ s time step. The phase shift due to EPLL digitalization and current control delay were compensated for via software.

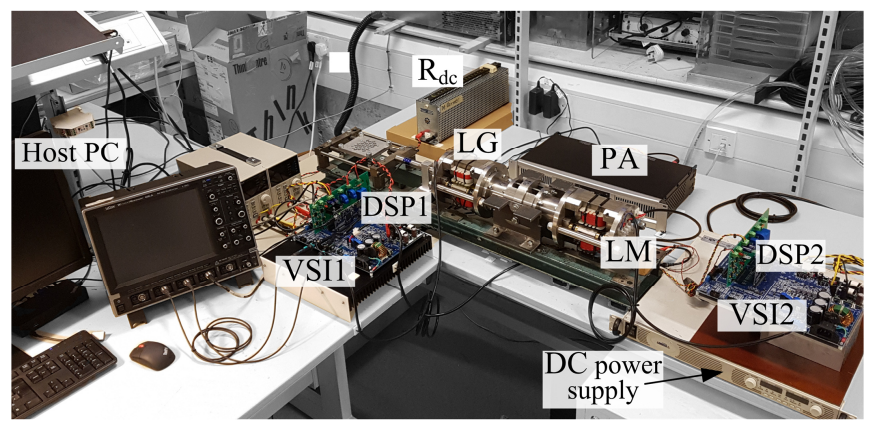

Fig. 7. Test-rig with back-to-back linear motor (LM) and LG.

Two setups were considered for feeding the prime-mover LM:

1) Hysteresis-current-controlled LM via a second TI VSI (VSI-2 + DSP-2) energized with a DC power supply.

2) LM driven by a Power Amplifier (PA) - PULSE TPA3400 directly driven by a function generator.

The LM current controller hysteresis band was deliberately set high (0.8A) to stress the robustness of the LG control.

Fig. 8 shows the experimental normalized stroke/frequency characteristics of the test rig at no-load in scenarios 1) and 2) compared to that of a force-driven system (identified by plotting the normalized stroke/current ratio).

Configuration 1) realizes a "Force-Driven" (FD) system with zero equivalent source impedance. Conversely, configuration 2) introduces significant "source" equivalent mechanical impedance which shifts the overall no-load resonance frequency from $36.5 \mathrm{~Hz}$ to $42.8 \mathrm{~Hz}$. Configuration 2) allows 
validation of the resonance tracking and tuning scheme for practical applications where the pressure wave source may introduce some unknown mechanical impedance.

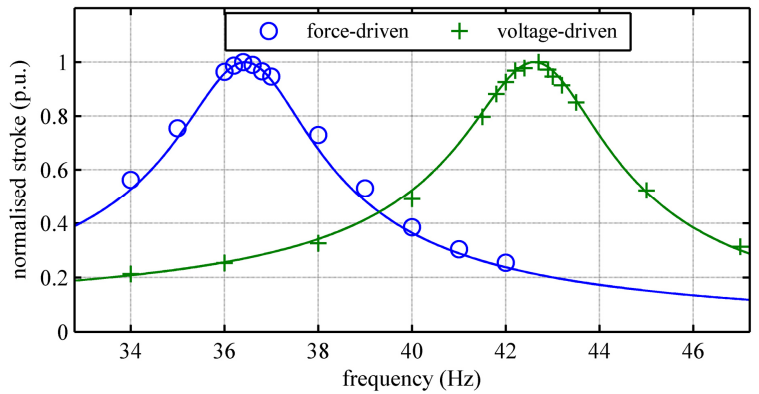

Fig. 8. Normalized stroke-frequency characteristics of the test-rig.

\section{A. Force-driven conditions}

\section{1) Start up}

Fig. 9 shows frequency $f$ and stroke amplitude $X$ from the PLL, dc power $p_{d c}, d$-axis reference current amplitude $I_{d}{ }^{*}$, and error $\varepsilon$ during the control initialization sequence.
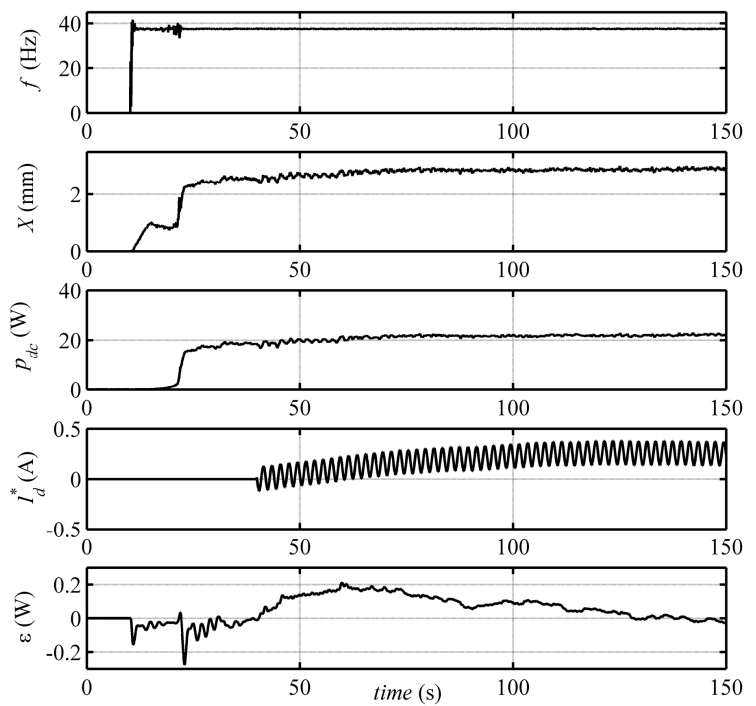

Fig. 9. Force-driven system: control initialization.

The $L G$ is run by the $L M$ at $37.5 \mathrm{~Hz}$, which is above the mechanical resonance frequency of $36.5 \mathrm{~Hz}$ as per markers "o" in Fig. 8. The LG current control is enabled with $I_{q}{ }^{*}=2 \mathrm{~A}$ while increasing the LM reference current to have a stroke of $3 \mathrm{~mm}$, which gives an overhead for transient overshoots. The value $I_{q}{ }^{*}=2 \mathrm{~A}$ allows some margin for $I_{d}{ }^{*}$ in closed-loop tests. The low-frequency modulation signal and closed-loop control are enabled at $t=40 \mathrm{~s}$. Initially, out-of-resonance error $\varepsilon$ is not zero as frequency is above the resonance value. Consequently, the control starts to reduce the error to zero by increasing $I_{d}{ }^{*}$. Steady-state and restored resonance are reached at $t \approx 130 \mathrm{~s}$.

\section{2) Open-loop results}

The system was then tested in open-loop conditions, i.e. with automatic tuning disabled, but with $d$ - axis current modulation enabled $\left(I_{\varepsilon}=0.12 \mathrm{~A}\right)$. This allows steady-state stroke and power modulation to be visualized and used to validate (16) and (21). The $q$-axis current amplitude was set to
$I_{q}{ }^{*}=2$ A with a LM current of $2.6 \mathrm{~A} \mathrm{pk}$ to achieve a $3 \mathrm{~mm}$ stroke at mechanical resonance $(36.5 \mathrm{~Hz})$ giving zero error $\varepsilon$.

Fig. 10 shows the response to a step increase of LM frequency from $36.5 \mathrm{~Hz}$ to $38.5 \mathrm{~Hz}$. Initially, the system runs at resonance and the stroke amplitude $X$ contains only small second-harmonic oscillations at $2 \times 0.5 \mathrm{~Hz}$, so $X_{\varepsilon}$ and $\varepsilon$ are both zero, according with (16) and (21). After the step to $38.5 \mathrm{~Hz}$, the frequency mismatch causes significant reduction of stroke, and triggers a visible power and stroke modulation of amplitude $2 \mathrm{~W}$ and $0.16 \mathrm{~mm}$ at $0.5 \mathrm{~Hz}$ (i.e. $\omega_{\varepsilon} / 2 \pi$ ). Therefore, error $\varepsilon$ increases and settles to the steady-state value $0.86 \mathrm{~W}$. Equations (16) and (21) give a theoretical amplitude $X_{\varepsilon} \approx 0.13$ $\mathrm{mm}$ and error $\varepsilon \approx 0.81 \mathrm{~W}$ : these are quite close to the experimental values and validate the theory in Section IV.
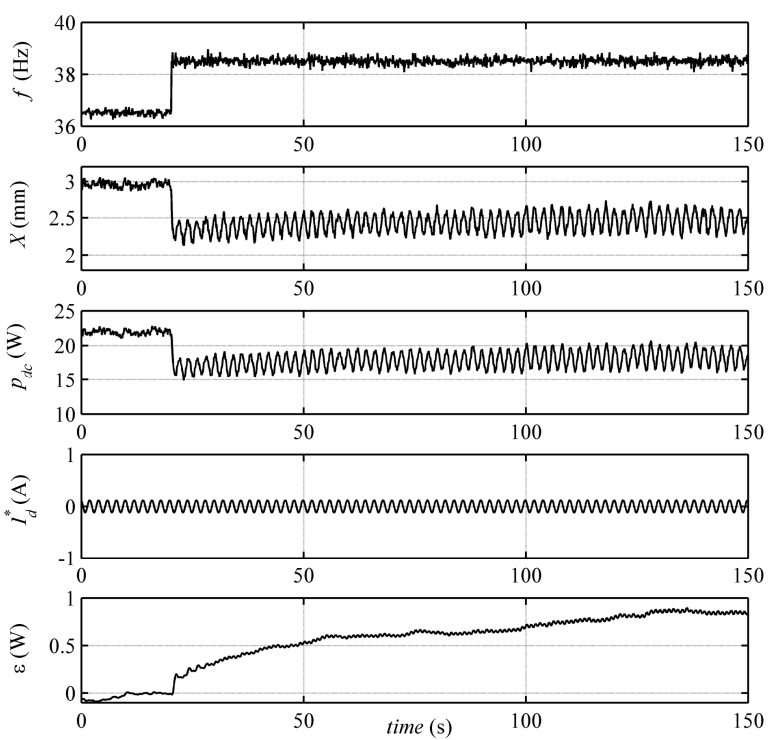

Fig. 10. Force-driven system open-loop response to a frequency step

Fig. 11 shows the steady-state instantaneous position and hysteresis-controlled LM current (proportional to driving force) along with its $1^{\text {st }}$-harmonic component, before (top) and after (bottom) the transient. The phase-shift between instantaneous position and LM-current $1^{\text {st }}$ harmonic changes from to $88.6^{\circ}$ (very close to the ideal $90^{\circ}$ resonance value) to $98.2^{\circ}$ showing that the LG is no longer at resonance. 

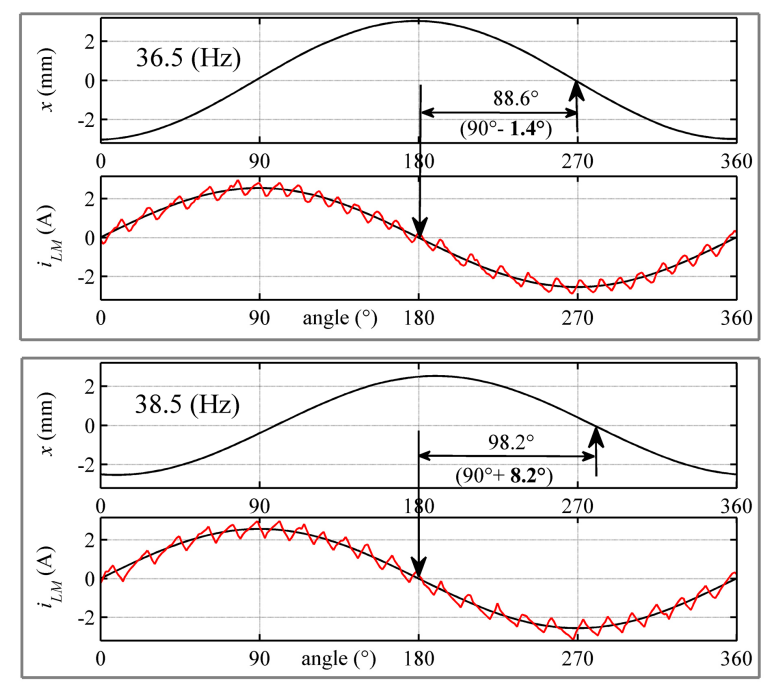

Fig. 11. Force-driven system, open-loop, steady-state position and LM current (total and $1^{\text {st }}$ harmonic) at $36.5 \mathrm{~Hz}$ and $38.5 \mathrm{~Hz}$.

\section{3) Closed-loop results}

Fig. 12 shows the closed-loop response under the same frequency step as in Fig. 10. After the stroke and power dips following the frequency step, error $\varepsilon$ increases according to the dynamics of the control loop. The PI controller reacts by applying a positive $d$-axis current $I_{d}{ }^{*}$ in order to generate positive electronic stiffness and increase the overall resonance frequency, so stroke and power can recover. After the transient, resonance conditions are restored and the stroke settles at $2.75 \mathrm{~mm}$, slightly below the initial value. This is due to the higher damping loss created by the higher frequency and velocity, the LM reference current and LG $I_{q}{ }^{*}$ set points being unvaried during the test. The resulting average $d$-axis current is $0.50 \mathrm{~A}$. These values are very close to $X_{1}=2.84 \mathrm{~mm}$ and $I_{d}$ res $=0.53$ A calculated by (7) and (17), and from simulation in Fig. 6. As predicted by (16), at "restored" resonance, stroke modulation $X_{\varepsilon}$ at $0.5 \mathrm{~Hz}$ disappears, confirming that at steady-state the current amplitude modulation does not disrupt prime-mover operation.
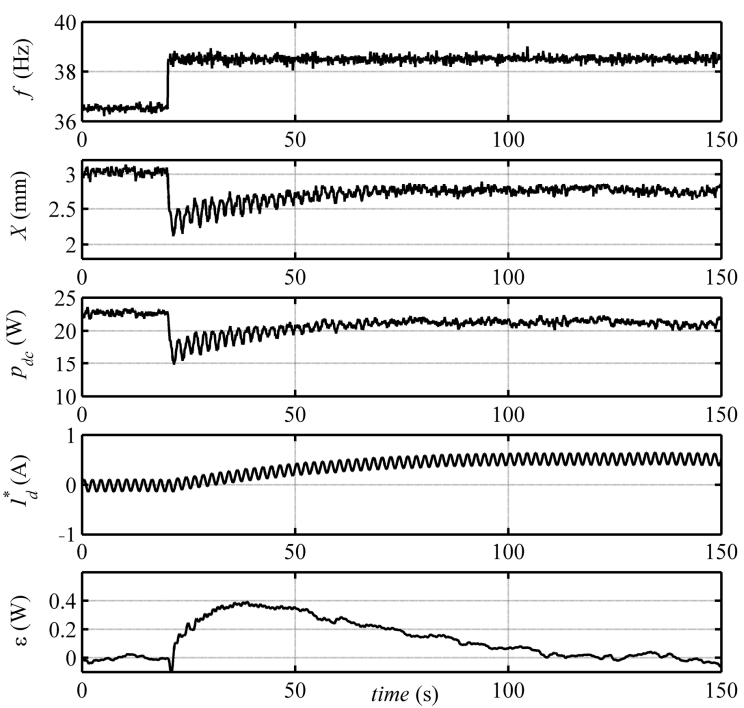

Fig. 12. Force-driven system closed-loop response to a frequency step.
Fig. 13 illustrates the final steady-state instantaneous position and hysteresis-controlled LM current (proportional to driving force) along with its $1^{\text {st }}$-harmonic component. The phase-shift between position and $1^{\text {st }}$-harmonic LM instantaneous current is now $93.2^{\circ}$ instead of the previous $98.2^{\circ}$ in Fig. 11-bottom. The lower deviation $\left(3.2^{\circ}\right)$ from $90^{\circ}$ proves that the control is able to bring the system back very close to resonance even if the frequency of $38.5 \mathrm{~Hz}$ is well above mechanical resonance.

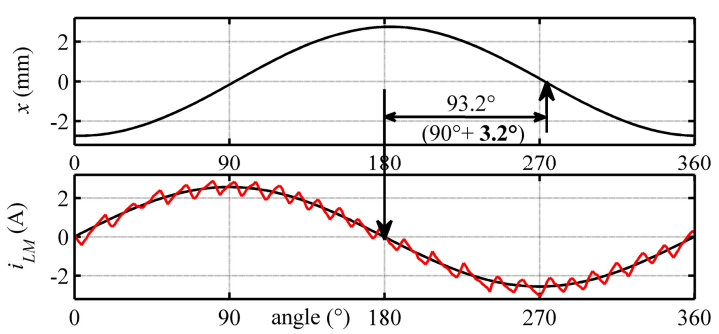

Fig. 13. Force-driven system, closed-loop, steady-state position and LM current (total and $1^{\text {st }}$ harmonic) at $38.5 \mathrm{~Hz}$ after frequency step.

\section{4) Effect of Joule and VSI losses}

The correction for Joule and VSI losses proposed in this paper is important when the system operates far from mechanical resonance and at moderate load levels. The effect of Joule and VSI losses is shown in Figs. 14 and 15 for closedloop operation at $41 \mathrm{~Hz}$ and $I_{q}{ }^{*}=1 \mathrm{~A}$. As the operating frequency is above the mechanical resonance frequency, the control forces $I_{d}{ }^{*}=1.32$ A to restore resonance. At $t=40 \mathrm{~s}$, the Joule and VSI loss correction is disabled, therefore triggering a transient in the error $\varepsilon$, leading to a decrease of $I_{d}{ }^{*}$ and stroke. Dc power $p_{d c}$ does not exhibit significant changes, but undesirable oscillations at $\omega_{\varepsilon}$ appear in stroke amplitude $X$. In addition, steady-state waveforms in Fig. 15 prove that resonance tracking accuracy deteriorates after disabling loss compensation. The phase-shift between instantaneous position and $1^{\text {st }}$-harmonic LM current is $86.5^{\circ}$ with loss compensation and $98.6^{\circ}$ without compensation: i.e. the deviation from the ideal $90^{\circ}$ value has grown from $3.5^{\circ}$ to $8.6^{\circ}$.
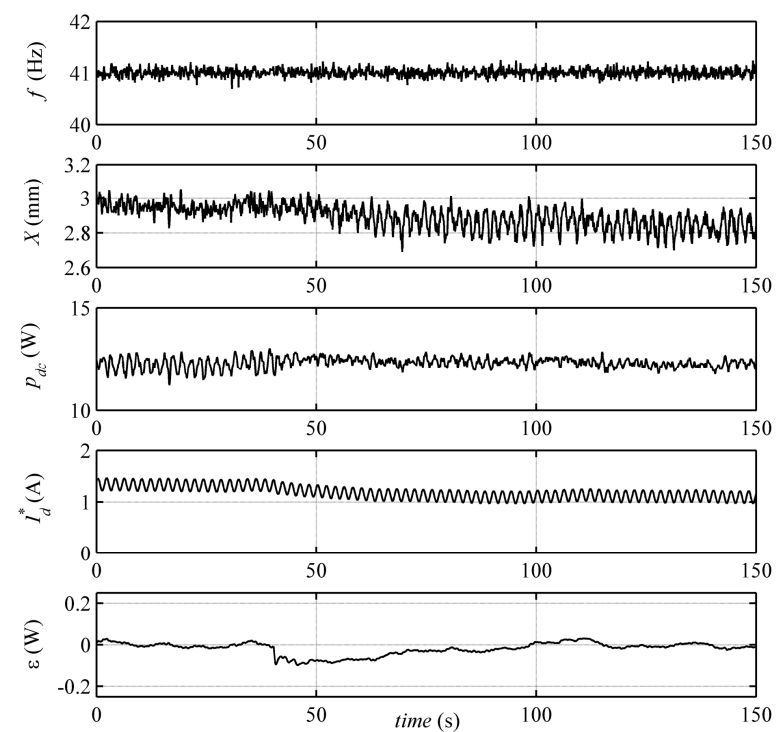

Fig. 14. Force-driven system closed-loop response after disabling 
Joule and VSI loss compensation.
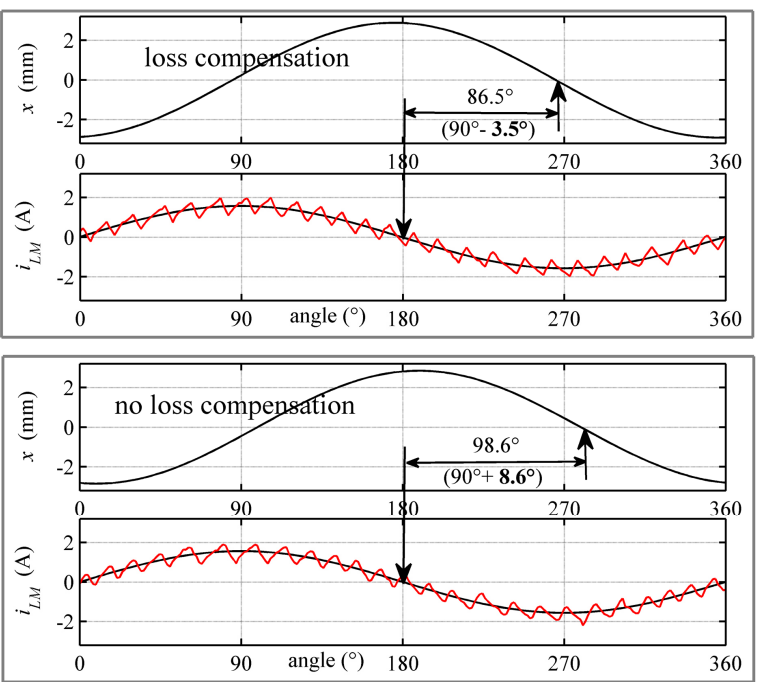

Fig. 15. Force-driven system closed-loop steady-state position and LM current (total and $1^{\text {st }}$ harmonic) with and without loss compensation.

\section{5) Results with distorted driving force}

The robustness of the control system to a distorted force excitation was tested by repeating the closed-loop response test in Fig. 14, while introducing a $25 \%$ third-harmonic component in the LM current. The results are shown in Fig. 16 and are very similar to those in Fig. 12 with monochromatic excitation. Fig. 17 shows the steady-state instantaneous position and LM current. The mechanical system filters out the distortion, and the steady-state stroke exhibits only a $2 \%$ third- harmonic component. The final steady-state phase shift between $1^{\text {st }}$-harmonic stroke and LM current component is $90.3^{\circ}$, showing that the system is able to restore resonance.
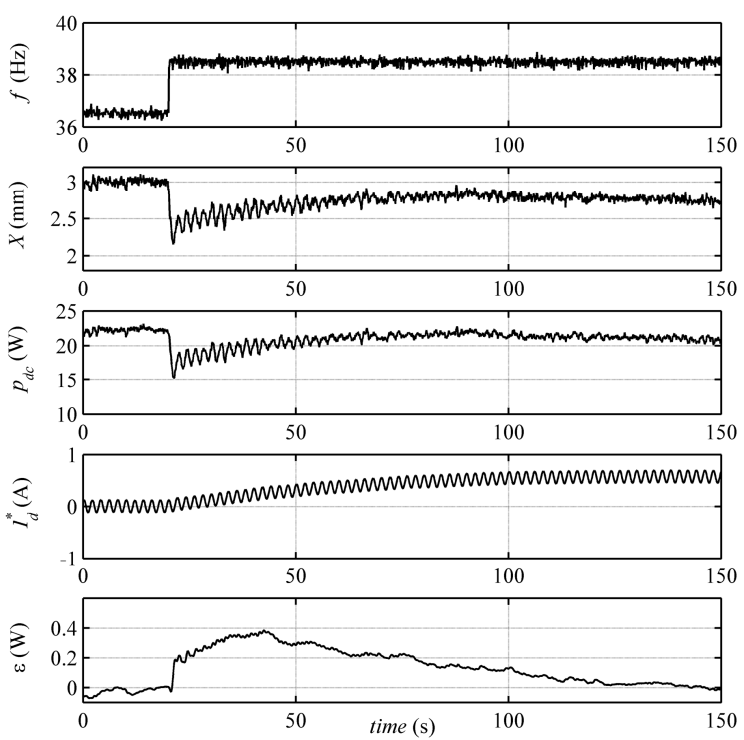

Fig. 16. Force-driven system, closed-loop response to a frequency step, under distorted force excitation ( $3^{\text {rd }}$ harmonic of $25 \%$ ).

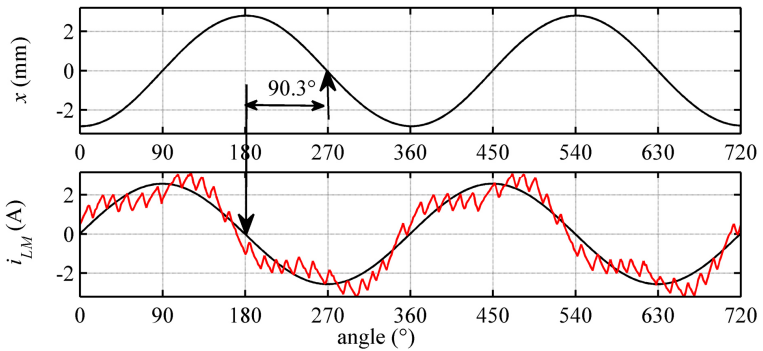

Fig. 17 Force-driven system, closed-loop, final steady-state position and LM current (total \& $1^{\text {st }}$ harmonic) in case of distorted LM current.

\section{B. Voltage-driven conditions}

The frequency step tests in Section VII-A were repeated by powering the LM with a PA driven by a function generator, with no current control for the motor. The reference resonance frequency at no load is now $42.8 \mathrm{~Hz}$ (curve markers "+" in Fig. 8) due to the significant prime-mover "source impedance" introduced by the electric dynamics of the LM stator.

Fig. 18 shows the response of the open-loop LG system to a frequency step-change from $42.4 \mathrm{~Hz}$ to $39.9 \mathrm{~Hz}$. Similarly to the previous test in Fig. 10, stroke and power decrease and exhibit visible amplitude modulation at $0.5 \mathrm{~Hz}$. Consequently, the out-of-resonance error $\varepsilon$ reduces from zero to the steadystate value $\varepsilon=-0.63 \mathrm{~W}$ proving that, unlike in force-driven conditions, the frequency of $39.1 \mathrm{~Hz}$ is far below the overall resonance frequency.

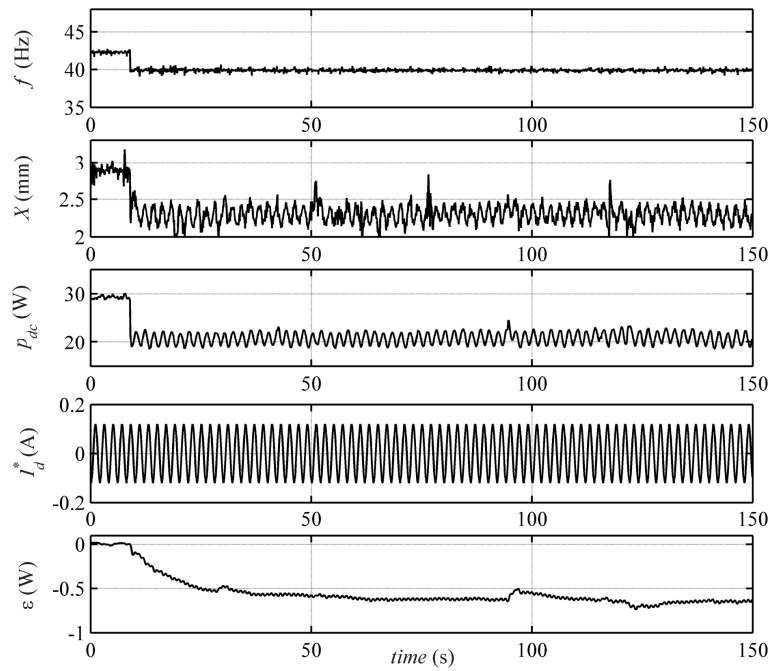

Fig. 18. Voltage-driven system: open-loop frequency step-response.

The same test was repeated with closed-loop control enabled. Results are shown in Fig. 19. After the initial dip in stroke and power caused by the frequency step, the PI controller reacts to error $\varepsilon$ and outputs a negative mean value for the $d$-axis current $I_{d}{ }^{*}$ in order to decrease the total stiffness and resonance frequency. The stroke and power recover and after $\approx 90 \mathrm{~s}$ the stroke settles at $2.9 \mathrm{~mm}$. The small increase compared to initial value of $2.8 \mathrm{~mm}$ is due to the lower damping force as the plunger is now moving at lower speed. 


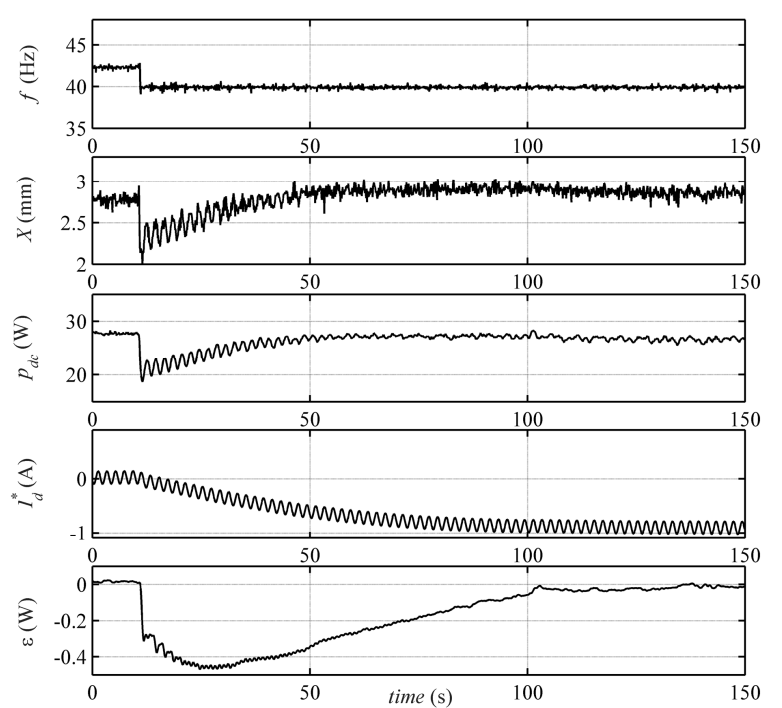

Fig. 19. Voltage-driven system: closed-loop frequency step-response.

\section{CONCLUSION}

An automatic resonance detection and electronic stiffness tuning strategy for reciprocating LGs was presented in this paper. Should the driving-force frequency deviate from the resonance value, the control adjusts the current component in phase with instantaneous position so as to vary the electronic stiffness and restore resonance. The resonance tracking method is based on the response of the LG to a quasi-static amplitude modulation of the current component in phase with instantaneous position. Compared to model-based techniques, the proposed method does not require knowledge of LG mechanical parameters and force-source mechanical impedance. The proposed analytic steady-state model exhibits only a $10 \%$ error, unlike previous modelling attempts in the Literature which show errors in excess of $80 \%$. The broad experimental validation proves that the control is able to restore resonance after changes in the force frequency even when the force-source introduces significant additional mechanical impedance. The tests also show that the implementation based on airgap power instead of dc power results in more accurate resonance tracking.

\section{REFERENCES}

[1] H. Polinder, B. C. Mecrow, A. G. Jack, P. G. Dickinson, M. A. Mueller, "Conventional and TFPM linear generators for direct-drive wave energy conversion," IEEE Trans. En. Conv., vol. 20, no. 2, pp. 260-267, June 2005.

[2] Ping Zheng, C. Tong, J. Bai, Bin Yu, Yi Sui, Wei Shi, "Electromagnetic design and control strategy of an axially magnetized permanent-magnet linear alternator for free-piston Stirling engines," IEEE Trans. Ind. Appl., vol.48, no. 6, pp. 2230-2239, Nov.-Dec. 2012.

[3] J. Xia, W. Li, R. Peng and H. Su, "Analysis on Axial End Flux Leakage and Resonance Characteristic of TFPM Linear Generator for Thermoacoustic Electric Generation System," IEEE Trans. Mag., vol. 52, no. 12, pp. 1-7, Dec. 2016.

[4] T. M. Lewis, A. von Jouanne, T. K. A. Brekken, "Per-unit wave energy converter system analysis," 2011 IEEE Energy Conversion Congress and Exposition, Phoenix, AZ, 2011, pp. 4123-4129.

[5] P. R. M. Brooking, M. A. Mueller, "Power conditioning of the output from a linear vernier hybrid permanent magnet generator for use in direct drive wave energy converters," IEE Proc. Gener. Transm. Distrib., vol.152, no. 5, pp. 673-681, Sept. 2005.

[6] V. Delli Colli, P. Cancelliere, F. Marignetti, R. Di Stefano, M. Scarano,
"A Tubular-Generator Drive For Wave Energy Conversion," IEEE Trans. Ind. Electron., vol. 53, no. 4, pp. 1152-1159, June 2006.

[7] R. Vermaak, M. Kamper, "Experimental Evaluation and Predictive Control of an Air-Cored Linear Generator for Direct-Drive Wave Energy Converters," IEEE Trans. Ind. Appl., vol. 48, no. 6, pp. 18171826, 2012.

[8] R. Vermaak, M. J. Kamper, "Design Aspects of a Novel Topology AirCored Permanent Magnet Linear Generator for Direct Drive Wave Energy Converters," IEEE Trans. Ind. Electron., vol. 59, no. 5, pp. 2104-2115, May 2012

[9] B. Yang, "Linear compressor control circuit to control frequency based on the piston position of the linear compressor". U.S. Patent US5947693A, 7 Sep 1999.

[10] J. Latham, M. L. McIntyre, M. Mohebbi, "Sensorless Resonance Tracking and Stroke Control of a Linear Vapor Compressor Via Nonlinear Observers," IEEE Trans. Ind. Electron., vol. 65, no. 5, pp. 3720-3729, May 2018

[11] T. Chun, J. Ahn, H. Lee, H. Kim, E. Nho, "A novel strategy of efficiency control for a linear compressor system driven by a PWM inverter," IEEE Trans. Ind. Electron., vol. 55, no. 1, pp. 296-301, Jan. 2008.

[12] J. Latham, M. L. McIntyre, M. Mohebbi, "Parameter Estimation and a Series of Nonlinear Observers for the System Dynamics of a Linear Vapor Compressor," IEEE Trans. Ind. Electron., vol. 63, no. 11, pp. 6736-6744, Nov. 2016.

[13] Z. Lin, J. Wang, D. Howe, "A resonant frequency tracking technique for linear vapor compressors," 2007 IEEE Int. Elect., Mach. Drives Conf. IEMDC'07, pp. 370-37, 2007.

[14] J. Shek, D. Macpherson, J. Xiang, "Reaction force control of a linear electrical generator for direct drive wave energy conversion," IET Ren. Pow. Gen., vol. 1, no. 1, pp. 17-24, 2007.

[15] J. K. H. Shek, D. E. Macpherson, M. A. Mueller, "Experimental verification of linear generator control for direct drive wave energy conversion," IET Ren. Pow. Gen., vol.4, no. 5, pp. 395-403, 2010.

[16] M. Iacchetti, R. Shuttleworth, M. Zhang, "Volt-Ampere Ratings in Electronically-Tuned Linear Alternators for Thermo-Acoustic Engines," IEEE Ren. Pow. Gen., vol. 12, no. 11, pp. 1256-1262, 2018.

[17] W. Li, K. T. Chau, T. W. Ching, C. Liu, "A Phase-Decoupled FluxReversal Linear Generator for Low-Speed Oscillatory Energy Conversion Using Impedance Matching Strategy," IEEE Trans. Ind. Electron., vol. 65, no. 9, pp. 7590-7599, Sept. 2018.

[18] E. Amon, T. Brekken, A. Schacher, "Maximum Power Point Tracking for Ocean Wave Energy Conversion," IEEE Trans. Ind. Appl., vol. 48, no. 3, pp. 1079-1086, 2012

[19] J. Park, B. Gu, J. Kim, H. Cho, “Active Phase Control for Maximum Power Point Tracking of a Linear Wave Generator," IEEE Trans. Pow. Electron., vol. 32, no. 10, pp. 7651-7662, 2017.

[20] K. Wang, J. Zhang, N. Zhang, "Acoustic matching of a traveling-wave thermoacoustic electric generator," Appl. Therm. Eng., vol. 105, no. 5, pp. 272-282, 2016.

[21] M. Iacchetti, M. Zhang, R. Shuttleworth, "Resonance Tuning in Linear Alternator Drives via Direct-Current Amplitude Modulation," in 23rd Int. Conf. Electr. Mach. (ICEM'2018), Greece, 2018.

[22] Z. Lin, J. Wang, D. Howe, "A Learning Feed-Forward Current Controller for Linear Reciprocating Vapor Compressors," IEEE Trans. Ind. Electron., vol. 58, no. 8, pp. 3383-3390, Aug. 2011.

[23] G. Masoud, "Linear and Pseudolinear Enhanced Phased-Locked Loop (EPLL) Structures," IEEE Trans. Ind. Electron., pp. 1464-1474, 2014.

[24] X. Xiao, X. Huang, Q. Kang, "A Hill-Climbing-Method-Based Maximum-Power-Point-Tracking Strategy for Direct-Drive Wave Energy Converters," IEEE Trans. Ind. Electron., vol. 63, no. 1, pp. 257267, Jan. 2016

[25] M. Jama, A. Wahyudie, "Online Damping Strategy for Controlling Heaving Wave Energy Converters Using Three-Phase Bridge Boost Rectifier," IEEE Access, vol. 5, pp. 7682-7691, 2017.

[26] E. Tedeschi, M. Molinas, "Tunable Control Strategy for Wave Energy Converters With Limited Power Takeoff Rating," in IEEE Transactions on Industrial Electronics, vol. 59, no. 10, pp. 3838-3846, Oct. 2012. 